\title{
LA PRÁCTICA REFLEXIVA: \\ UNA ALTERNATIVA PARA LA \\ ENSEÑANZA DEL DIBUJO ANALÍTICO
}

REFLECTIVE PRACTICE: AN ALTERNATIVE FOR TEACHING ANALYTICAL DRAWING

Dora del Carmen Segrera Tapia
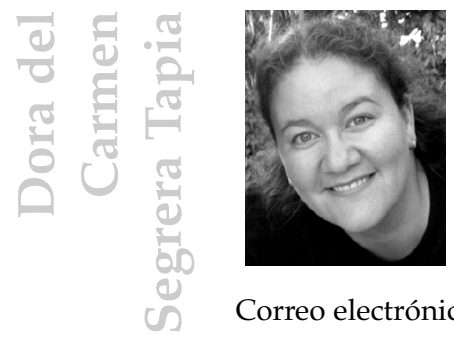

Maestría en Educación, Universidad Panamericana. Licenciatura en Diseño Gráfico, Universidad Iberoamericana. Ha dedicado los últimos 17 años a la docencia en dichas instituciones, especializándose en las diferentes disciplinas del dibujo: analítico, natural, figura humana, técnico, introspectivo, en movimiento, entre otros.

Correo electrónico: [dora.segrera@gmail.com].

\section{RESUMEN}

El presente artículo retoma algunos de los postulados básicos de la práctica reflexiva —orígenes, momentos de la reflexión en la acción, prácticum reflexivo, actores del proceso, diálogo reflexivo-y plantea su relación con la enseñanza del dibujo analítico, a nivel universitario, promotor del pensamiento plástico tridimensional y de su correcta comunicación dentro del proceso de diseño, mediante estrategias didácticas que permiten al docente, desde su papel formador, ayudar al estudiante para que su aprendizaje adquiera sentido, impulsándolo a desarrollar las conexiones conceptuales y prácticas necesarias en el ejercicio de su profesión. 
Palabras clave: práctica reflexiva; reflexión en la acción; diálogo; dibujo analítico.

\section{ABSTRACT}

This article revisits some of the basic tenets of reflective practice -its origins, the moments of reflection in action, reflective practicum, actors in the process, reflective dialogue - and raises its relationship with education at university level, analytical drawing, three-dimensional plastic promoter of thought and its proper communication within the design process by teaching strategies that allow the teacher from his formerrole, helping students to acquire learning context, urging him to develop the conceptual connections and practices necessary in the exercise of their profession.

Key words: reflective practice; reflection in action; dialogue; analytical drawing.

\section{INTRODUCCIÓN}

Mi incursión en el campo de la práctica reflexiva se sustenta en la búsqueda de una respuesta pertinente para un cuestionamiento fundamental: cómo promover la formación de profesionales reflexivos, específicamente a partir de la asignatura de dibujo analítico. Dicha interrogante nace de la observación repetida, a lo largo de mi experiencia docente, de una problemática común entre los estudiantes de diseño a nivel licenciatura: la falta de motivación del alumno hacia el aprendizaje, la falta de transversalidad entre los contenidos de las materias, y la falta de transferencia entre lo aprendido en la carrera y el ámbito profesional.

Ante tales premisas, surge la inquietud de diseñar estrategias orientadas a promover la motivación y propiciar el aprendizaje significativo, para contribuir a la formación de alumnos capaces no solo de seguir instrucciones, sino de saber hacer y, más aún, de saber lo que están haciendo, para llevar ese conocimiento a nuevos campos; estudiantes, en síntesis, capaces de construir su propio conocimiento, que serán, por tanto, profesionales reflexivos. 
Contrario a lo que se piensa, el dibujo para el diseño dista mucho de ser el dibujo libre y expresivo del artista; en realidad, es un dibujo más estructurado y metodológico. El dibujo analítico —más allá de capacitar al alumno en el dominio de instrumentos, habilidades o técnicas de la materia- busca desarrollar en el estudiante el pensamiento plástico, tridimensional y lógico, la capacidad de pensar en papel, de exponer sus ideas, su concepción del mundo y los objetos, plasmándolos en un espacio bidimensional, utilizando un código claro, preciso y explícito de comunicación gráfica, que pueda comprenderse fácilmente por cualquier sujeto involucrado en el proceso de diseño.

Por otro lado, entendiendo la universidad como un espacio dinámico, intelectual, comunitario, cultivador del saber y preservador de la cultura -donde profesores y estudiantes comparten una visión común del hombre y el mundo-, puede inferirse que la esencia misma de la universidad está conformada por la relación dialógica entre sus principales actores: el docente (que debe poseer carácterísticas específicas de su ser y actuar como persona, profesionista y educador) y el estudiante (que debe reunir condiciones especiales, como disponibilidad, búsqueda de explicaciones, solicitud de ayuda y apertura a la reflexión para, mediante ellas, tender a la excelencia, tanto académica y profesional, como personal y social). Los objetivos y contenidos de las materias que integran el currículum de los diversos programas académicos deben ser congruentes con la búsqueda de esta excelencia.

\section{LA PRÁCTICA REFLEXIVA}

La práctica reflexiva representa una opción metodológica importante, tanto para la actuación docente, como para la consecución del aprendizaje, pues el conocimiento y la aplicación de las fases del proceso reflexivo — estado de duda, estado de búsqueda y decisión-y la ejecución de los momentos que lo constituyen - reflexión en la acción, reflexión sobre la acción y reflexión para la acción- favorecen el desarrollo de profesionales reflexivos, capaces de promover relaciones dialógicas, propiciando así un proceso dinámico de reflexión simultánea; un espacio abierto en el que comparten conocimientos, inquietudes y problemáticas, comunes e individuales, donde todos se beneficien de las experiencias de los demás, generando un mutuo 
enriquecimiento académico y haciendo de la reflexión un principio de actuación permanente.

\section{John Dewey: el pensamiento reflexivo}

El filósofo de la educación, John Dewey propone en su libro How we think (1989: 21), que la mejor manera de pensar se denomina pensamiento reflexivo, es decir, el «tipo de pensamiento que consiste en darle vueltas a un tema en la cabeza y tomárselo en serio con todas sus consecuencias». Sostiene también que el pensamiento reflexivo implica necesariamente dos fases: un estado de duda, de vacilación, de perplejidad, de dificultad mental, en el que se origina el pensamiento, y un acto de búsqueda, de caza, de investigación, para encontrar algún material que esclarezca la duda.

Al enfrentarse a una situación confusa, una persona puede eludirla, fantasear con dominarla o enfrentarse a ella realmente; en este último caso, comienza a reflexionar. El acto completo de la actividad reflexiva está limitado por una situación pre-reflexiva, que plantea el problema a resolver y una post-reflexiva, donde la duda se ha disipado; en la zona intermedia, se sitúan propiamente las fases o los aspectos del pensamiento reflexivo: sugerencias, intelectualización, hipótesis, razonamiento y comprobación (Dewey, 1989: 102-103).

Para Dewey, el pensamiento reflexivo debe constituir un objetivo de la educación pues, aunque el pensamiento otorga al hombre la capacidad de actuar con un objetivo consciente - anticipándose a las situaciones con sistemas creadores o modificadores, y dotando a las cosas de significación-, este no se realiza automáticamente y requiere una cuidadosa orientación educativa pues,

[...] aun cuando no podemos aprender ni enseñar a pensar, podemos aprender cómo pensar bien, sobre todo cómo adquirir el hábito general de reflexión (1989: 47).

Para ello, los docentes deben ser conscientes de las fuerzas operativas de las que pueden servirse: experiencias, esperanzas, deseos e intereses de los estudiantes, para comprenderlas y dirigirlas hacia la formación de hábitos reflexivos. 


\section{Donald Schön: el profesional reflexivo}

El filósofo Donald Schön ha retomado las ideas de Dewey, centrando su trabajo y su investigación en los problemas del aprendizaje de las organizaciones y de la eficacia profesional. En sus libros The reflective practitioner: How professionals think in action $y$ Educating the reflective practitioner, postula la «reflexión en la acción» como el medio para potenciar el aprendizaje, y el desarrollo de habilidades de pensamiento y la resolución de problemas. Esta corriente surge como una alternativa frente a los limitados alcances de la racionalidad técnica (que dejaba en la ciencia y el conocimiento teórico, todo el peso de la acción racional); ante esta postura, Schön defiende la idea de que los problemas no siempre se presentan como estructuras bien organizadas y, por otro lado, que las personas se enfrentan a ellos de maneras diferentes, en función de la propia experiencia. $\mathrm{Y}$ son precisamente estas zonas indeterminadas, las áreas centrales en la práctica profesional.

Una preocupación cada vez más constante es

[...] la falta de conexión existente entre la idea de conocimiento profesional que prevalece en las escuelas profesionales y aquellas competencias que se les exigen a los prácticos en el terreno de la realidad (Schön, 1987: 23).

En cuanto a la preparación profesional, Schön (1987: 27) afirma que la «profesionalización» de la Universidad, gestada a partir de principios del siglo XX, «significó la sustitución del arte por el conocimiento sistemático, preferentemente científico» $\mathrm{y}$, ante los resultados de este pragmatismo sistémico, hoy los formadores han empezado a ver de nuevo al «arte como un componente esencial de la competencia profesional».

En este sentido, el estudio del dibujo analítico es un claro ejemplo de la integración holística de estas corrientes, pues retoma la metodología y el rigor científico, combinándolos en un marco natural de observación de la realidad, añadiendo un toque de creatividad en la resolución de problemas concretos. Schön (1987: 33) propone su propia definición del arte profesional, entendiéndolo como los tipos de competencia que los prácticos muestran algunas veces en situaciones 
de la práctica y que resultan singulares, inciertas y conflictivas. En el estudio de las artes, el énfasis no se centra en el conocimiento, sino en el aprender haciendo (descrito por John Dewey y retomado por Schön), como «la disciplina básica o inicial», pues está enfocado a saber cómo emprender el logro de los fines, incidiendo en la aplicación de lo aprendido.

Schön (1987: 29) apoya la idea diciendo que

[...] los estudiantes aprenden mediante la práctica de hacer o ejecutar aquello en lo que buscan convertirse en expertos, y se les ayuda a hacerlo así por medio de otros prácticos más veteranos que les inician en las tradiciones de la práctica.

Sin embargo (y siguiendo la línea de Dewey), aclara que «no se puede enseñar al estudiante lo que necesita saber, pero puede guiársele». A partir de esta premisa, se gesta el concepto del prácticum reflexivo: "prácticas que pretenden ayudar al estudiante a adquirir las formas de arte que resultan esenciales para ser competente en las zonas indeterminadas de la práctica» (Schön, 1987: 30), donde el diálogo entre estudiante y profesor (más cercano a un tutor, según describe Schön), se convierte en un elemento indispensable, pues entremezcla la descripción de la práctica con la propia ejecución.

\section{Estructura de la práctica reflexiva}

Sara Galbán (2006: 109), citando a Zeichner, afirma que la acción reflexiva supone la consideración activa, persistente y cuidadosa de cualquier creencia o forma supuesta de conocimiento a la luz de sus fundamentos y de las consecuencias que promueve. También menciona que la práctica reflexiva se convierte en una actitud que, siguiendo a Bourdieu, se llama habitus, entendido como el conjunto de esquemas que posee la persona en un momento específico de su vida y que la llevan a fomentar la instauración de esquemas reflexivos, surgidos de la regularización y sistematización del pensamiento espontáneo. 
Concluye Galbán (2006: 137) que

[...] la práctica reflexiva busca potenciar la capacidad que tiene el ser humano de reflexionar, para convertir esta operación de la inteligencia en una actitud y en un habitus profesional.

Esta «costumbre de reflexionar» se presenta como una constante tanto en profesores como en estudiantes, dentro como fuera del aula, a nivel profesional como personal; es decir, se convierte en una forma de vida, que promueve el desarrollo de la persona humana en todos sus ámbitos, relaciones y potencialidades.

\subsection{Conocimiento en la acción}

Para entender cómo surge una práctica reflexiva, es necesario conocer algunos conceptos ligados a esta forma de aprender. Schön (1987: 33) retoma de Ryle la idea de que «inteligente» no puede definirse en términos de «intelectual», ni «saber cómo» en términos de «saber qué» (pensar en lo que hago no implica "pensar qué hacer y a la vez hacerlo»). De Polanyi adopta, para explicar este concepto, el término de conocimiento tácito. Y de Barnard los procesos no lógicos, es decir, «aquellos juicios, decisiones y acciones eficientes que realizamos de un modo espontáneo, sin ser capaces de establecer las reglas o los procedimientos que seguimos».

Schön delinea las propiedades del conocimiento en la acción; la observación y la reflexión sobre las propias acciones permiten describir el conocimiento tácito (aquellas ejecuciones espontáneas que, paradójicamente, no pueden explicitarse de modo verbal), concluyendo que

[...] la actividad de conocer sugiere la cualidad dinámica de conocer en la acción que, cuando la describimos, se convierte en conocimiento en la acción (1987: 36).

\subsection{Reflexión en la acción y reflexión sobre la acción}

Todas las experiencias de la vida poseen un factor sorpresa y se puede responder a este dejándolo pasar, sin prestarle atención, o mediante la reflexión; en este segundo caso, puede hacerse de dos maneras: reflexionando en la acción o sobre la acción. 
Podemos reflexionar sobre la acción, retomando nuestro pensamiento sobre lo que hemos hecho para descubrir cómo nuestro conocimiento en la acción puede haber contribuido a un resultado inesperado. Podemos hacerlo así una vez que el hecho se ha producido, ya tranquilamente, o podemos realizar una pausa en medio de la acción [...], en cualquier caso, nuestra reflexión carece de una conexión directa con la acción presente.

De un modo alternativo, podemos reflexionar en medio de la acción, sin llegar a interrumpirla. En una acción presente [...], nuestra acción de pensar sirve para reorganizar lo que estamos haciendo mientras lo estamos haciendo [...] en casos como este, estamos reflexionando en la acción (Schön, 1987: 36-37).

Estas formas de reflexionar constituyen la base de la práctica reflexiva, pues ya sea que este análisis reflexivo se dé en el propio momento en el que se desarrolla la acción, o una vez que esta ha pasado; ambos casos implican la puesta en marcha de procesos mentales a partir de situaciones no determinadas, donde el patrón de indagación de este tipo de procesos surge como una secuencia de momentos «por ensayo y error», métódicamente ordenados.

\subsection{Reflexión sobre la reflexión en la acción}

Schön lleva la reflexión un paso más allá: cuando somos capaces de verbalizar lo que hemos resuelto mediante la acción reflexionada. La reflexión sobre la reflexión, propicia un diálogo entre pensamiento y acción, permitiendo volver a pensar acerca de una determinada experiencia, lo que puede dar pie a consolidar la comprensión del problema o, más aún, imaginar una mejor o más completa solución.

Igual que el conocimiento en la acción, la reflexión en la acción es un proceso que podemos llevar adelante sin ser capaces de decir lo que estamos haciendo [...] una cosa es ser capaz de reflexionar en la acción y otra muy distinta es ser capaz de reflexionar sobre nuestra reflexión en la acción, de manera que produzcamos una buena descripción verbal de ella, e incluso otra cosa es ser capaz de reflexionar acerca de la descripción resultante (Schön, 1987: 40). 
Brubacher, Case y Reagan (2005: 35), al adaptar el pensamiento de Schön, distinguen en la práctica reflexiva tres tipos de reflexión: reflexión en la práctica; reflexión sobre la práctica, y reflexión para la práctica:

Tanto la reflexión en la práctica como la reflexión sobre la práctica son, esencialmente, de índole reactiva y se distinguen, sobre todo, por el momento en el que la reflexión se lleva a cabo: en la primera, se realiza durante la práctica [...] y en la segunda, la reflexión se lleva a cabo después de un hecho específico. Por otra parte, y según Killion y Todnem, la reflexión para la práctica es «el resultado deseado de los dos tipos de reflexión previos. Reflexionamos no tanto para volver al pasado o tomar conciencia del proceso metacognitivo que estamos experimentando [...] sino para orientar nuestra acción futura, vale decir, por un propósito moral práctico» (Brubacher, 2005: 37-38).

\section{Práctica profesional y prácticum reflexivo}

\section{Una práctica profesional es}

[...] la competencia de una comunidad de prácticos que comparten, en palabras de John Dewey, las tradiciones de una profesión. Comparten convenciones de acción que incluyen medios, lenguajes e instrumentos distintivos (Schön, 1987: 40).

Los miembros de una profesión tienen diferencias individuales, pero comparten un tronco común de conocimiento profesional. El conocimiento en la acción de un profesional surge dentro del contexto social e institucional de una comunidad de prácticos; el conocimiento en la práctica se ve afectado (positiva o negativamente) por dicho espacio común del saber.

Una persona puede aprender la práctica de varias maneras: por sí misma, como aprendiz de otro o participando de un prácticum. Un prácticum es «una situación pensada y dispuesta para la tarea de aprender una práctica», se recrea un contexto similar al mundo real de la práctica, donde los estudiantes pueden: 
[...] aprender haciéndose cargo de proyectos que simulan y simplifican la práctica, relativamente libre de las presiones, las distracciones y los riesgos que se dan en el mundo real al que, no obstante, el prácticum hace referencia (Schön, 1987: 46).

El estudiante que ingresa en un prácticum resuelve tareas básicas - como evaluar una práctica competente, valorar su posición ante ella, elaborar un mapa del camino a seguir, adaptarse a las demandas, aprender «la práctica del prácticum» (instrumentos, métodos, proyectos y posibilidades) - $\mathrm{y}$, a todo esto, añadir su propia imagen personal. El trabajo del prácticum combina el aprender-haciendo de los alumnos, la interacción con los tutores y compañeros, y el aprendizaje experiencial. Este trabajo se produce bajo la dirección de un práctico veterano, que funge como un tutor cuyas funciones son: demostrar, aconsejar, plantear problemas y criticar; los alumnos frecuentemente resultan tan valiosos unos para los otros como el tutor mismo (Schön, 1987: 46).

\section{Actores de la práctica reflexiva y su relación dialógica}

En el ámbito de la práctica reflexiva es fundamental entender a los actores que la sustentan - docentes y discentes- y a la relación indisoluble, dialógica y de mutuo crecimiento, que surge entre ellos. Una práctica verdaderamente reflexiva involucra la participación activa tanto de maestros como de alumnos, en un proceso que integra elementos de distintos ámbitos, donde la experiencia manifiesta de ambos actores resulta indispensable para su éxito y operatividad.

Brubacher (2005: 35) argumenta que el trabajo diario del maestro consiste en emitir juicios, tomar decisiones reflexivas y conscientes, justificando estas decisiones y acciones, mediante un proceso racional que considera y evalúa las alternativas, bajo un determinado criterio, más que considerar a la enseñanza como un arte o un conjunto de habilidades técnicas.

Perrenoud (2007: 164) sostiene que, para ser un practicante reflexivo, no basta con tener una formación de alto nivel, con un grado académico o excelentes recursos intelectuales. Las universidades estén pobladas de eruditos que no saben enseñar y que no se plantean nada 
sobre el tema. Por otro lado, afirma que un «formador reflexivo» no forma en la reflexión por el solo hecho de tomar él mismo una postura reflexiva, sino que hace falta una intención orientada al entrenamiento para la reflexión y el análisis, y unos dispositivos centrados en diferentes ámbitos de conocimientos y de competencias.

Ludmerer, citado por Brubacher (2005: 35), concluye que la educación debe capacitar al estudiante para aprender durante toda su vida y no, simplemente, dominar la información y las técnicas del presente. Para lograr esto, dice que «los alumnos deben ser educandos dinámicos e independientes y dedicarse a resolver problemas, en lugar de ser meros receptores pasivos de información».

De este modo, la enseñanza reflexiva va de la mano con la educación permanente. El papel del profesor adquiere una relevancia distinta a la tradicionalmente aceptada, pues el protagonismo y la superioridad docentes, dan paso a una nueva dimensión académica: el maestro como formador e impulsor de la autonomía del estudiante ante su propio aprendizaje. Esta tarea implica, muchas veces, salir de los esquemas conocidos y cómodos para adentrarse en un terreno de mayor reto y responsabilidad, amén de comprometer el propio actuar con un proceso reflexivo cotidiano, no siempre placentero, pero sin duda enriquecedor.

Por otra parte, Perrenoud (2007: 18) destaca que algunos estudiantes buscan en la enseñanza lo que ya no se ofrece: ortodoxia y conocimientos prácticos, dejando a un lado la formación reflexiva que esta propone; las razones con las que explica este fenómeno son, por un lado, que han desarrollado una relación con el saber y el oficio que no les invita a la reflexión y, además, que los objetivos de una educación reflexiva no se han explicado suficientemente, para que el discente sea capaz de escoger otra orientación o abandonar imágenes estereotipadas de su oficio. Así, afirma que:

Formar un practicante reflexivo es ante todo formar a un profesional capaz de dominar su propia evolución, construyendo competencias y saberes nuevos o más precisos a partir de lo que ha adquirido y de la experiencia. El saber analizar es una condición necesaria, pero no suficiente para la práctica reflexiva, que exige una postura, una identidad y un habitus específico (Perrenoud, 2007: 23). 
De este modo, el papel del estudiante adquiere también un nuevo sentido. No basta ya con el tránsito de la actitud pasiva a la activa, el apendizaje reflexivo invita a revisar la acción, observándola, cuestionándola y manipulándola, para dirigirla hacia nuevos panoramas, en una visión no-lineal del saber, que permite volver sobre los pasos, analizar las variables, plantear alternativas de solución, transferir conocimientos entre diferentes disciplinas y ámbitos de la vida. De nuevo, esta visión implica un desafío a la educación, pues muchas veces el estudiante ha sido condicionado a recibir, sin mucho esfuerzo, un conocimiento digerido, fácil y amoldado.

Brockbank y McGill (2008: 57) resaltan que los especialistas en educación, como Bloom, señalan tres dominios de aprendizaje, que abarcan los tres aspectos: cognitivo (saber), contativo (hacer) y afectivo (sentir). Un gran reto de la educación superior consiste, precisamente, en que se ha privilegiado al conocimiento teórico, conceptual y memorístico, dejando de lado los otros dos dominios involucrados, es decir, los conocimientos de carácter procedimental y actitudinal-valoral. Brockbank y McGill, como muchos otros, apremian a voltear la mirada hacia esta situación y sostienen que cuestiones como la inteligencia emocional, la criticidad, el diálogo reflexivo y la integración del entorno, son componentes necesarios para aprender. El modelo integrado del aprendizaje que ellos sugieren, señala la valoración de los tres dominios y del contexto sociopolítico.

Perrenoud (2007: 43), por su parte, considera que para que un estudiante llegue a convertirse en practicante reflexivo, debe renunciar a sobrecargar el currículo académico inicial de conocimientos disciplinares y metodológicos, dejando tiempo y espacio para un procedimiento clínico, la resolución de problemas y el aprendizaje práctico de la reflexión profesional. Afirma que más que suministrar al estudiante todas las respuestas posibles, la formación reflexiva multiplica las ocasiones para que los estudiantes, en las aulas y en las prácticas, se forjen esquemas generales de reflexión y regulación.

Desde esta perspectiva, la relación enseñante-estudiante debe surgir a través del diálogo reflexivo, dinámico, bidireccional. Brockbank y McGill (2008: 69) plantean que la comprensión y práctica de dicho diálogo constituyen la responsabilidad emergente de los profesores de 
enseñanza superior y de sus instituciones. Enfatizan que el carácter y la forma del discurso producido en el diálogo reflexivo poseen consecuencias en el modo de relación de los profesores con los alumnos en las universidades; sostienen que el procedimiento unidireccional del saber es antiético y que hace falta una interacción bidireccional para que el alumno pueda convertirse en una persona críticamente reflexiva.

Sostienen que la transición del alumno a la autonomía y la independencia del aprendizaje no brota de manera individual, sino como un proceso social de interdependencia - tanto entre profesores y alumnos, como entre los estudiantes mismos-, pues para que el diálogo reflexivo sea eficaz, debe existir una relación bidireccional entre las partes, entendida como una correspondencia afectiva, que puede darse mediante la facilitación, tomando como centro de atención al aprendiz y no a la transmisión de contenidos. Proponen dos elementos necesarios para este diálogo reflexivo: emoción y transparencia (Brockbank y McGill, 2008: 69-70). En este sentido, la práctica reflexiva es un sistema de educación personalizada, pues incide directamente en la formación de las notas constitutivas de la persona: singularidad, autonomía y apertura.

De este modo, podemos inferir que una práctica verdaderamente reflexiva involucra la participación activa tanto de profesores como de estudiantes, en un proceso dialógico que integra elementos de distintos ámbitos, donde la experiencia manifiesta y la voluntad dispuesta de ambos actores resulta indispensable para su éxito y operatividad. Es interesante hacer notar que, en esta metodología, ningún actor resulta más importante que el otro y, sin embargo, la ausencia de cualquiera de ellos desequilibraría la ecuación.

\section{LA PRÁCTICA REFLEXIVA EN EL ÁMBITO DEL DIBUJO ANALÍTICO}

Schön ejemplifica la reflexión en la acción a partir del proceso de diseño. Entiende el diseño como una forma de creación, en la que el practicante (diseñador) construye una realidad, a partir de elementos aislados, que manipula y transforma gracias a procesos de reflexión: 
Un diseñador realiza una imagen, una representación de algo que quiere convertir en realidad, haya sido o no concebido originalmente en términos visuales, espaciales o plásticos. En su sentido más amplio, el diseño implica complejidad y síntesis... un diseñador juega con las variables, reconcilia los valores en conflicto y transforma los impedimentos. Se trata de un proceso en el que, aun a sabiendas de que unos productos diseñados pueden ser mejores que otros, no existe una única respuesta válida (Schön, 1987: 49).

Retoma también la visión de John Dewey sobre el diseñador como «alguien que transforma situaciones indeterminadas en determinadas», de nuevo mediante un proceso de análisis y crítica que privilegia el papel del diálogo reflexivo como medio para lograr el fin:

Partiendo de situaciones indeterminadas, mal definidas, complejas e incoherentes, los diseñadores construyen e imponen una coherencia propia. Más tarde, descubren consecuencias e implicaciones, algunas involuntarias, de sus construcciones, que aprecian y evalúan. El análisis y la crítica juegan un papel relevante dentro de un proceso más amplio. Su proceso de diseño viene a ser una trama de pasos medidos con antelación y de consecuencias e implicaciones descubiertas sobre la marcha que a veces llevan a la reconstrucción de la coherencia inicial, en definitiva, un diálogo reflexivo con los componentes de una determinada situación (Schön, 1987: 50).

Dentro de este proceso de diseño, se sitúa específicamente el ámbito del dibujo como herramienta indispensable para plasmar las ideas conceptuadas. Schön (1987: 54) afirma que «hablar y dibujar son formas paralelas de diseñar $y$, en conjunto, constituyen [...] el lenguaje de diseño». El dibujo analítico se coloca dentro del universo del dibujo, como una fusión entre lo técnico y lo artístico, pues requiere tanto de metodologías sistemáticas para el análisis volumétrico estructural, como del uso del apunte (bosquejo, boceto, esbozo), la composición, la luz y el color como elementos de representación de realidades visibles o imaginadas con fines comunicativos.

Aunque el estudio del dibujo incluye elementos de tipo declarativo $\mathrm{y}$ actitudinal, se trata de un conocimiento marcadamente procedimental, pues es un conocimiento práctico basado en la acción y la ejecución ordenada de actividades para llegar a una meta; estos pasos incluyen la apropiación de datos, la ejecución, automatización y el perfeccionamiento 
del procedimiento. Por lo tanto, su enseñanza no consiste en dotar al estudiante de un patrón o una ruta ideal ya probada y garantizada, sino guiarlo a través de su aprendizaje, permitiéndole cometer errores, sopesar opciones, enfrentar problemas e investigar posibles soluciones. Involucra una serie de estrategias y acciones encaminadas a adquirir niveles de dominio — no solo de habilidades específicas-, sino de una completa y organizada forma de pensar y hacer, habituando al estudiante a adoptar una actitud reflexiva frente a su actuar, reflexión que surge antes, durante y después de que este ocurre.

El dibujo analítico, por lo tanto, se convierte en un ejemplo idóneo de aplicación de la práctica reflexiva, pues los principios constitutivos de la reflexión se integran naturalmente a la manera de enseñar, comprender, practicar y hacer más eficientes los procesos de enseñanza de este tipo de dibujo, que busca promover en el alumno habilidades de visualización espacial, análisis-síntesis, resolución de problemas, soltura y rapidez, entre otras.

\section{Naturaleza del dibujo analítico}

Si atendemos al lugar que ocupa dentro de las ciencias del conocimiento, el dibujo analítico puede situarse dentro de la Didáctica de las Matemáticas, en la ciencia específica de la Geometría. Esta ubicación dentro de las Matemáticas, y no en la Didáctica de la Educación Artística como podría pensarse, se encuentra plenamente justificada por la naturaleza de sus contenidos y la proyección de sus objetivos. El fin último de esta materia no es la expresión, ni la sensibilización, sino la visualización espacial, su representación bidimensional y la comunicación conceptual.

Alan Pipes (2008: 12), en su libro Dibujo para diseñadores, entiende al dibujo como un medio necesario en el proceso de pensamiento, pues «el acto de dibujar estimula otro pensamiento, y este requiere otro dibujo y así sucesivamente»; defiende al boceto - herramienta clave de pensamiento en el dibujo analítico- como «una vaga idea de lo que un diseño podría ser, aun antes de que exista». 
El dibujo analítico, se fundamenta en un tema medular: el estudio de la perspectiva. En palabras de Pipes (2008: 45), «la perspectiva práctica es una forma de introducir distorsiones sistemáticas en los dibujos para representar la realidad». La definición de la perspectiva se sustenta en un principio básico de observación: los objetos parecen disminuir su tamaño conforme se alejan del observador y sus líneas paralelas parecen converger hacia determinados puntos en el horizonte. Este principio, de tan fácil detección en la práctica actual, requirió de muchos siglos en la historia del arte para sistematizarse y llevarse al campo de la bidimensionalidad pictórica. El retrato de una realidad tridimensional en un espacio de dos dimensiones, tal y como esta era apreciada por el ojo humano, no se logró hasta la época del Renacimiento.

El dibujo analítico posee carácterísticas diferentes al dibujo artístico, del cual, sin embargo, no puede separarse del todo, ya que principios como la composición, la armonía, la iluminación o la expresión, también se encuentran contenidos en su enseñanza, aunque con una presencia menos protagónica: la esencia del dibujo analítico radica, como se mencionó anteriormente, en la visualización espacial, la representación bidimensional y su correcta comunicación. Si la práctica reflexiva promueve - mediante el diálogo- el razonamiento lógico, la exploración, la observación y la construcción reflexiva, partiendo de una realidad concreta, el dibujo se torna un espacio particularmente eficaz para su aplicación, pues el pretexto temático del bocetaje de objetos de diseño y la práctica continua, propician un ambiente adecuado para el intercambio de ideas, la creación de un lenguaje común, la generación de procesos de pensamiento, el análisis de nuevas alternativas de solución, la definición de estilos personales, el desarrollo de competencias específicas y la aplicación de los conocimientos adquiridos en otras áreas del saber, por mencionar algunas de sus bondades.

\section{Estrategias didácticas para la promoción de profesionales reflexivos a partir del dibujo analítico}

Galbán (2006: 263) sintetiza las estrategias didácticas como el

[...] conjunto de métodos, técnicas y procedimientos que buscan optimizar y eficientar el proceso de enseñanza aprendizaje, con miras a facilitar la 
comprensión y apropiación del contenido tanto por parte del profesor como de los estudiantes.

Afirma que puede haber tantas estrategias como profesores reflexivos existan, y estas serán efectivas mientras promuevan el diálogo y favorezcan la reflexión del estudiante mediante la interacción directa con el objeto de estudio, pues le permitirán reunir experiencias y tener elementos para apropiarse del contenido.

Monereo (2007: 17) afirma que el factor reflexivo es determinante en la definición de una estrategia de aprendizaje, diferenciándola de la mera comprensión y aplicación de una técnica de estudio, pues implica enseñar al alumno a decidir sus actos y modificarlos de manera consciente, para orientarlos hacia el objetivo buscado.

Insistiendo en la relevancia del saber procedimental para el dibujo analítico, debe evitarse proporcionar al alumno una ruta ideal, permitiéndole enfrentar problemas e investigar posibles soluciones, induciendo la reflexión y el análisis continuo sobre las actuaciones, enseñando los contenidos mediante actividades con condiciones determinadas, como las propuestas por Antoni Zabala (1998: 84-85) en La práctica educativa: cómo enseñar:

- Las actividades deben partir de situaciones significativas y funcionales, para que el contenido se aprenda con la capacidad de utilizarlo cuando sea conveniente.

- La secuencia debe contemplar actividades que presenten los modelos de desarrollo del contenido del aprendizaje, con una visión completa de los pasos que lo componen.

- Las actividades de enseñanza aprendizaje deben ajustarse al máximo a una secuencia clara, con un orden que siga un proceso gradual -en este caso, para los contenidos específicos del dibujo analítico- del más sencillo al más complejo.

- Se requieren actividades con ayudas de diferente grado y práctica guiada. La estrategia más apropiada consiste en suministrar ayudas e ir retirándolas progresivamente; el alumno irá asumiendo el control de la ejecución.

- Del mismo modo, deben practicarse actividades de trabajo independiente, en las que el alumno muestre su competencia en el 
dominio del contenido aprendido para, posteriormente, ser capaz de aplicarlo en contextos diferenciados.

El dibujo analítico, en tanto asignatura curricular universitaria, propone una secuencia temática que va desde los conceptos más básicos al planteamiento de problemáticas de diseño, en donde el alumno debe emplear los conocimientos previamente adquiridos y las competencias desarrolladas para generar posibles alternativas de solución. La metodología se basa en la práctica cotidiana de los conceptos que se estudian; el conocimiento no se adquiere por memorización, sino por movilización de los procesos de pensamiento, a partir del planteamiento de problemas, la indagación, la reflexión, el análisis, la repetición y la comprobación de resultados. Cada estrategia didáctica planeada debe presentar, de manera sencilla y ordenada, los siguientes elementos:

- Tema: contenido programático que se pretende abarcar durante la sesión.

- Competencia: desempeño eficaz que se busca trabajar con la estrategia, interviniendo de manera integrada conocimientos, habilidades y actitudes, aplicables en diferentes contextos.

- Objetivo: meta, fin o intención a lograr mediante la estrategia, claramente definida.

- Enunciado de la estrategia: proposición que describe el plan de acción para lograr el objetivo (esta evidenciará la aplicación de la práctica reflexiva para su cumplimiento).

- Duración: tiempo total en que se llevará a cabo la estrategia.

- Desarrollo: integrado por dos secciones: el desglose de las actividades de la estrategia (explicadas paso a paso) y el tiempo programado para cada una.

- Recursos: conjunto de material didáctico, instrumentos, objetos y enseres necesarios para el desarrollo de la estrategia.

- Evaluación de la estrategia: producto que permite constatar si se han cumplido y en qué medida, los objetivos planteados (también este apartado reflejará explícitamente el uso de la práctica reflexiva).

Todo el trabajo realizado por el alumno en clase se concentra en una bitácora (elaborada con formato y material especiales), recurso 
gráfico que concentra todos los temas y ejercicios de clase (notas, procesos, dibujos), lo que permite al alumno tener a la mano los conocimientos previos, corroborar métodos y resolver sus propias dudas. Los reforzadores de aprendizaje, sugeridos como ejercicios a realizar fuera del aula tras cada sesión, se trazan en hojas sueltas (por la logística de evaluación de dichas tareas por parte del profesor) llamadas láminas, que se organizan y recogen al final del curso en otra bitácora. Ambos instrumentos proporcionan una valiosa herramienta para la reflexión, pues las láminas reunidas no solo proporcionan conocimientos de manera aislada, sino que promueven en el estudiante habilidades para volver sobre sus pasos, reforzar procesos, hacer relaciones y modificar su actuar en todo momento.

De igual modo, se sugiere al profesor llevar un diario de clase, en el que registre todo lo observado: el avance y desarrollo de sus alumnos (individual y grupalmente), la participación y actitud del estudiante durante la actividad, las problemáticas detectadas, las estrategias eficaces. Este instrumento permite al docente reflexionar cotidianamente alrededor de su propia práctica, para dirigir sus acciones y sustentar sus decisiones.

\section{REFLEXIONES FINALES}

Las tendencias educativas actuales apuntan cada vez más al equilibrio entre los actores del proceso de enseñanza aprendizaje, dejando atrás el protagonismo docente, pero valorando el papel del profesor como facilitador y elemento necesario para el aprendizaje. Desde tal perspectiva, el prácticum reflexivo se antoja como un lugar armónico donde se privilegia el proceso mismo, pero apoyado en los actores que lo ejecutan: el diálogo como promotor del mutuo aprendizaje, logra el equilibrio perfecto entre los vértices de la tríada del proceso de enseñanza aprendizaje (contenido educativo-educador-educando) o, visualizándolo gráficamente, lleva a dicho triángulo de una concepción vertical, a un planteamiento horizontal de las partes involucradas, donde ninguno de los elementos es más importante que el resto, pero todos resultan indispensables para mantener a la figura equilibrada. La práctica reflexiva, desde sus inicios, mantiene este equilibrio entre los actores del proceso, situando en el centro la relación 
dialógica que se da entre ellos, forjando, a partir de la reflexión en la acción, sobre la acción y para la acción, una interacción complementaria, de mutuo crecimiento y no de competencia.

La práctica reflexiva — más que una eficiente metodología académicase presenta aquí como una herramienta de vida, pues sienta las bases de un pensamiento ordenado, que vuelve sobre sus pasos y provoca un discernimiento sobre el actuar, activando procesos que reorganizan lo que se hace y redirigen el propio caminar. Más aún, en un ámbito prospectivo, la reflexión adquiere su razón de ser al enfocarse hacia la mejora permanente, como sucede en los procesos de evaluación. Es decir, la reflexión adquiere sentido no solo por el análisis de la acción realizada, sino por las propuestas que este análisis genera y el seguimiento que se hace de las mismas, elementos sin los cuales la simple introspección resultaría estéril.

El docente, como facilitador, acompaña al estudiante que, mediante este diálogo, potencia sus capacidades, no solo cognitivas. Estas se convierten en una suerte de pretexto para ayudarlo a desarrollar habilidades mentales como el juicio crítico, el razonamiento, el pensamiento divergente, la autorreflexión y la metacognición, en un proceso donde aprendizaje y reflexión se fusionan volviéndose elementos inseparables. Por supuesto, esta práctica lleva implícito el compromiso, esfuerzo y entrega de ambas partes, planteando un camino complejo, difícil y en ocasiones extenuante, pero al mismo tiempo, lleno de satisfacciones ante la contemplación de los logros, pues la ejercitación y la aplicación de la práctica reflexiva como un habitus conduce a la formación de un estilo de vida más congruente, seguro y productivo; en suma, más pleno.

En este sentido, el dibujo analítico resulta un campo fértil para la aplicación de la enseñanza reflexiva, pues su naturaleza funcional -más que expresiva - y su finalidad como promotor de pensamiento plástico, visualización espacial y correcta comunicación de ideas, encuentran en el «aprender haciendo», la práctica repetida y la reflexión cotidiana, el camino óptimo para el aprendizaje. Por tratarse de un taller siempre dinámico, la relación que se genera entre profesor y estudiantes - $\mathrm{y}$ entre los discentes mismos- promueve el razonamiento, la argumentación, la deducción, la aplicación y el intercambio 
de ideas, todos ellos elementos de la práctica reflexiva, en el momento mismo en que se realiza la acción.

Como materia, el dibujo analítico lleva una línea metodológica que permite una seriación integrada y progresiva de los contenidos programáticos, de tal suerte que, al final del curso - y con la plena conciencia de que una materia semestral no puede abarcar todas las nociones existentes de una disciplina-, el conocimiento adquirido y las habilidades desarrolladas por cada estudiante, les permitan continuar de manera personal y autónoma los procesos iniciados, promoviendo así una verdadera formación reflexiva permanente.

De la experiencia misma de sistematizar y aplicar estrategias didácticas enfocadas a enseñar y aprender el dibujo analítico, mediante la práctica reflexiva, pueden rescatarse varios aprendizajes:

- La reflexión personal en, sobre y para la acción permite, como docente, tomar conciencia del propio proceder, reorientar los pasos y encontrar nuevas soluciones.

- El diálogo reflexivo proporciona un reflejo del actuar, pues el punto de vista del otro se convierte en el punto de partida, el medio y el fin de la propia reflexión, facilitando también una correspondencia afectiva más enriquecedora. Dicho diálogo propicia también cambios sobre la marcha que, sin descuidar los contenidos programáticos de la materia, cubren las áreas de mayor interés detectadas, promoviendo además, la investigación, la inferencia y la experimentación del estudiantado.

- La reflexión favorece una dinámica grupal sana e integrada, pues los estudiantes mantienen una buena motivación y disposición hacia la construcción conjunta de los conocimientos, mediante elementos como la creatividad, la analogía y el juego.

- La aplicación de ejercicios que llevan al análisis crítico de situaciones prácticas, permite realizar aplicaciones transversales de los contenidos de esta disciplina hacia otras materias y plantear escenarios (hipotéticos y reales) hacia los cuales lograr la correcta transferencia de los conocimientos recién adquiridos. 
- El uso de herramientas de registro, como la bitácora y los reforzadores, impulsa la construcción reflexiva con una visión holística de lo aprendido, abriendo un espacio de práctica que facilita, entre otras cosas, la retención de métodos, el análisis de procesos, la observación estructurada y el registro de los avances.

- La evaluación centrada en los logros personales y el desarrollo individual — sin perder de vista los objetivos del curso-, si bien implica más esfuerzo y compromiso, también redunda en una mayor satisfacción de ambas partes ante la evidencia recabada.

$\mathrm{Al}$ exponer estos resultados, busco transmitir la idea de que la enseñanza reflexiva funciona, de que es una herramienta viva que nos permite, como docentes, desarrollar una práctica profesional más ética, armónica, funcional, equilibrada y productiva. Los estudiantes, por su parte, obtienen también de la práctica reflexiva grandes beneficios al hacer de la reflexión en, sobre y para la acción, una habilidad continua, adquieren conciencia de su propio aprendizaje, desarrollan competencias significativas para su profesión, perfeccionan sus procesos de pensamiento y toman decisiones mucho más acertadas. Los alcances de esta metodología son ilimitados pues, como todo proceso, se encuentra en constante construcción, deconstrucción y reconstrucción, pero es precisamente esta característica de transformación permanente, la que aporta sentido a su uso y sustenta su permanencia en el ámbito educativo. 


\section{REFERENCIAS BIBLIOGRÁFICAS}

BROCKBANK, A. y McGILL, I., Aprendizaje reflexivo en la educación superior, $2^{\mathrm{a}}$ ed., Morata, Madrid, 2008, 311 p.

BRUBACHER, J.W.; CASE, Ch.W. y REAGAN, T.G., Cómo ser un docente reflexivo, $2^{a}$ ed., Gedisa, Barcelona, 2005, 173 p.

DEWEY, J., Cómo pensamos: nueva exposición de la relación entre pensamiento y proceso educativo, Paidós, Barcelona, 1989, 249 p.

DÍAZ-BARRIGA ARCEO, F., Enseñanza situada: vínculo entre la escuela y la vida, Mc Graw-Hill/Interamericana, México, 2010, 171 p.

GALBÁN LOZANO, S.E., La enseñanza reflexiva de la didáctica en la formación de pedagogos, Tesis doctoral, Universidad de Barcelona, Facultad de Pedagogía, Barcelona, 2006, 350 p.

MONEREO, C. (coord.), Estrategias de enseñanza y aprendizaje: formación del profesorado y aplicación en la escuela, $1^{\mathrm{a}}$ ed., Graó/Colofón, México, 2007, 191 p.

PERRENOUD, Ph., Desarrollar la práctica reflexiva en el oficio de enseñar: profesionalización y razón pedagógica, $3^{\mathrm{a}}$ ed., Graó, Barcelona, 2007, 224 p.

PIPES, A., Dibujo para diseñadores, $1^{\text {a }}$ ed. esp., Blume, Barcelona, 2008, 224 p.

SCHÖN, D.A., La formación de profesionales reflexivos: hacia un nuevo diseño de la enseñanza y el aprendizaje en las profesiones, $1^{a}$ ed., Paidós, Barcelona, 1987, 310 p.

ZABALA, A., La práctica educativa. Cómo enseñar, $4^{\mathrm{a}}$ ed., Graó, Barcelona, 1998, 233 p. 